\title{
PRIMARY DIFFUSE LEPTOMENINGEAL GLIOMATOSIS
}

\author{
André Leite Gonçalves', Marcelo Rodrigues Masruha', Henrique Carrete Jr², \\ João Norberto Stávale ${ }^{3}$, Nasjla Saba da Silva ${ }^{4}$, Luiz Celso Pereira Vilanova ${ }^{7}$
}

Glioma arising primarily from the leptomeninges is extremely rare and often diagnosed only in postmortem examination. It is thought to derive from heterotopic nests of neuroglial tissue within the leptomeninges, which are present in $1 \%$ of necropsies. Primary leptomeningeal glioma can be in the form of a solitary tumour or a diffuse tumour involving intracranial or spinal cord leptomeninges'.

We report a case of primary diffuse leptomeningeal gliomatosis (PDLG) established by meningeal biopsy.

\section{CASE}

A 13-year-old boy initiated symptoms at age of 7 years, with a partial complex seizure. He remained stable until the age of 8 , when he presented another seizure. At this time he was admitted in coma into the critical care unit, and underwent treatment for viral encephalitis with acyclovir for 14 days. He was discharged with clinical improvement. Afterwards he presented recurrent headaches.

Two years later he was admitted with headache, neck stiffness, drowsiness and vomiting. At this time an intracranial hypertension syndrome was identified. The cranial MRI showed a communicating hypertensive hydrocephalus that required a ven- triculoperitoneal shunt. He was stable up to the age of 11, when a dysfunction of the ventriculoperitoneal shunt was identified and the shunt was changed. At this time etiologic investigation for chronic meningitis was initiated. Histoplasma capsulatum was considered the probable agent, because of his positive epidemiology and he underwent treatment with fluconazole. He was treated for two years and referred to our service.

On admission at our hospital, at age of 13 , he complained of sporadic headache, which was being treated with sodium valproate and fluconazole, with no seizures in the last year. During the investigation, different lumbar punctures were performed with elevated protein levels (up to $5.4 \mathrm{~g} / \mathrm{L}$ ), but glucose levels were normal. CSF white blood cells were up to $80 / \mathrm{mm}^{3}$. No neoplastic cells were detected. Acid fast bacilli smear, culture and tuberculosis DNA polymerase chain reaction (PCR) technique were all negative. Mycological analysis for histoplasmosis, paracoccidioidomycosis, aspergillosis and candidiasis were all negative. Immunological reactions to syphilis, toxoplasmosis, cytomegalovirus, cysticercosis and brucellosis were negative. Electroencephalograms revealed a generalized asynchronous slowing of background waves.

Cranial CT showed diffuse calcifications in the basal cisterns suggesting some consequence of inflammatory process (Fig 1A).
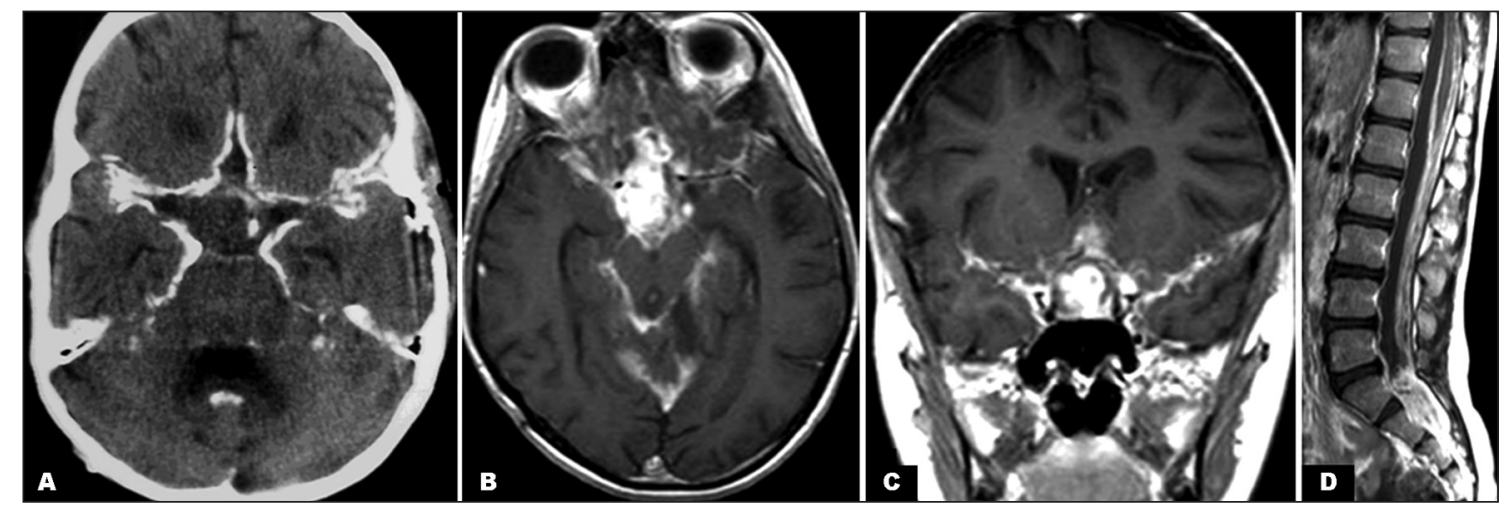

Fig 1. (A) Axial CT demonstrates calcification along the surfaces of frontal and temporal lobes, suprasellar region and cerebellar vermis. Postgadolinium axial (B) and coronal (C) T7-weighted MR images. Note extensive enhancement of basal cisterns extending into lateral fissures after contrast ( $B$ and $C$ ). In $C$, note thickening of the left lateral ventricle ependyma. Sagital T7-weighted postcontrast (D) MR images of lumbar spine demonstrate markedly leptomeningeal enhancement.

\section{GLIOMATOSE LEPTOMENINGEA PRIMÁRIA DIFUSA}

Universidade Federal de São Paulo (UNIFESP), São Paulo SP, Brazil: 'Department of Neurology and Neurosurgery; ${ }^{2}$ Department of Radiology; ${ }^{3}$ Department of Pathology; ${ }^{4}$ Department of Paediatrics / Paediatric Oncology Institute.

Received 12 June 2007, received in final form 23 October 2007. Accepted 24 November 2007.

Dr. André Leite Gonçalves - Division of Child Neurology / Departamento de Neurologia e Neurocirurgia - Rua Botucatu 720 - 04023-900 São Paulo SP - Brasil.E-mail: goncalvesnp@yahoo.com.br 

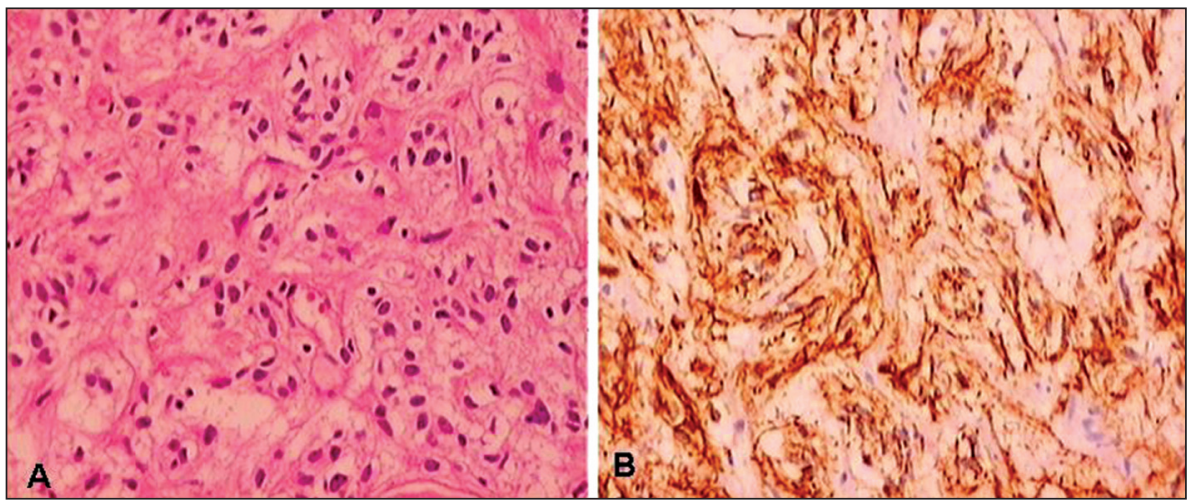

Fig 2. (A) HE, Meningeal biopsy showing astrocytic glial proliferation in the sub-arachnoid space with a; (B) GFAP, positive stain for glial fibrillary acidic protein, conclusive for a low-grade glioma.

Non-enhanced cranial and spinal cord MRI was normal. Postgadolinium images revealed diffuse enhancement in cerebral sul$\mathrm{ci}$, cerebellar vermis, basal, pontine and ambient cisterns (Fig 1B and 1C) and in the spinal cord (Fig 1D) with no evidence of intraparenchymal involvement.

A meningeal biopsy was performed and histologic examination revealed an astrocytic glial proliferation in the subarachnoid space (Fig 2A) with a positive stain for glial fibrillary acidic protein (Fig 2B), conclusive for a low-grade glioma. Based on this result, associated with clinical and radiological data, a diagnosis of primary leptomeningeal gliomatosis, low grade glioma type was made.

During this period, he was assessed by the oncology team and initiated temozolomide cycles, completing 22 cycles associated to neuroaxis radiotherapy. He progressed with scarce seizures, using valproate (Depakote) and acetazolamide (Diamox) and presented longitudinal nasal hemianopsy that evolved to complete involvement of the visual field. After the temozolomide cycles, initiated chemotherapy sessions with ciisplatin and etoposide. At 16 years of age, he did not resist to a pulmonary infection and died after a cardiac and -respiratory arrest.

\section{DISCUSSION}

PDLG is a rare disease. It is characterized by a glioma, usually an astrocytoma, which arises in the leptomeninges of the brain or spinal cord with no involvement of intraparenchymatous tissue ${ }^{2}$. Two anatomical and clinical forms have been described: a nodular form and a diffuse form ${ }^{3,4}$, which corresponds to our observation.

Debono et al. ${ }^{3}$ recently reviewed the literature on this subject and found only 30 cases previously reported. The mean age of clinical onset was 35 years, ranging from 9 to 71 years, with no sex predominance. The neurological symptoms are mainly related to intracranial hypertension ( $83.8 \%$ of cases), followed by multiple cranial nerve palsy (35.5\%) and epilepsy (29.03\%). Other signs include hemiparesis and tetraparesis, behavioural changes, confusion, visual loss and gait disturbances, back pain, meningeal signs and coma.
CSF findings usually show an increase in protein levels with low or moderate pleocytosis ${ }^{3}$. Repeated CSF examinations are usually negative for malignant cells; moreover, distinguishing chronic aseptic meningitis from leptomeningeal carcinomatosis or gliomatosis may be difficult since malignant cells can be misinterpreted as benign macrophages. In assessing patient's risk, immunocytochemistry with appropriate immunostains for GFAP or cytokeratins and epithelial membrane antigen (EMA) may be helpful ${ }^{4-7}$. However immunocytochemistry is not used in routine cytological evaluation but frequently performed in the CSF after a positive brain biopsy.

Neuroradiological findings include ventriculomegaly with diffuse or focal leptomeningeal contrast enhancement. The spinal cord is predominantly involved, either associated or not with contrast enhancement of the basal cisterns, cerebellum, brainstem and convexity ${ }^{3}$. A pattern of non-enhancing nodular lesions on the leptomeninges was described. In our patient these findings are similar to those reported in the literature with diffuse enhancement in cerebral sulci, cerebellar vermis, basal, pontine and ambiens cisterns and in the entire spinal cord with no evidence of intraparenchymal involvement ${ }^{1,3}$.

Surgical meningeal biopsy is necessary in cases of chronic aseptic meningitis with areas of contrast enhancement to determine the primary cause of this disease. In this case, meningeal biopsy was decisive to determine the etiology of the disease and to establish the proper treatment.

Differential diagnoses include carcinomatous meningitis and autoimmune and inflammatory processes affecting the meninges, such as tuberculous meningitis ${ }^{3,8-10}$. Treatment is intended to reduce the intracranial pressure. Some patients undergo a CSF diversion procedure in the form of ventriculoperitoneal or another alternative route shunting ${ }^{3}$.

Craniospinal radiation therapy and chemotherapy can be employed. Beauchesne et al. ${ }^{9}$ obtained a complete clinical and radiological remission for 15 months after a 
course of corticospinal tract radiation therapy and chemotherapy. In our patient, remission occurred after complete treatment, that is, thirty months later.

The prognosis is generally poor and survival depends on tumour grade and patients do not survive as long as ours. In this case, the long evolution, for nine years, is probably due to the fact it is a low-grade glioma.

\section{REFERENCES}

1. Kastenbauer S, Danek A, Klein W, et al. Primary diffuse leptomeningeal gliomatosis: unusual MRI with non-enhancing nodular lesions on the cerebelar surface and spinal leptomeningeal enhancement. J Neurol Neurosurg Psychiatry 2000;69:385-388.

2. Asworth B, Gordon A. Leptomeningeal gliomatosis. J Neurol Neurosurg Psychiatry 1994;57:471-473.

3. Debono B, Derrey S, Rabehenoina C, Proust F, Freger P, Laquerrière A. Primary diffuse multinodular leptomeningeal gliomatosis: case report and review of the literature. Surg Neurol 2006;65:273-282
4. Wechsler LD, Gross RA, Miller DC. Meningeal gliomatosis with "negative" CSF cytology: the value of GFAP staining. Neurology 1984; 34: 1661-1665.

5. Bilic M, Welhs CT, Rumboldt Z, Hoda RS. Disseminated primary diffuse leptomeningeal gliomatosis: a case report with liquid based and conventional smear cytology. Cytojournal 2005;2:1-5.

6. Dietrich PY, Aapro MS, Rieder A, Pizzolato GR. Primary diffuse leptomeningeal gliomatosis (PDLG): a neoplastic cause of chronic meningitis simulating tuberculous meningitis. J Neuroncol 1993;15:275-283.

7. Thomas JE, Falls E, Velasco ME, Zaher A. Diagnostic value of immunocytochemistry in leptomeningeal tumor dissemination: a report of two cases. Arch Pathol Lab Med 2000;124:759-761.

8. Barborie A, Dunn EM, Bridges LR, Bamford JM. Primary diffuse leptomeningeal gliomatosis predominantly affecting the spinal cord: case report and review of the literature. J Neurol Neurosurg Psychiatry 2001; 70:256-258.

9. Beauchesne P, Pialat J, Duthel R, et al. Aggressive treatment with complete remission in primary diffuse leptomeningeal gliomatosis. J Neuroncol 1998;37:161-167.

10. Rees JH, Balakas N, Agathonikou A, et al. Primary diffuse leptomeningeal gliomatosis simulating tuberculous meningitis. J Neurol Neurosurg Psychiatry 2001;70:120-122. 